\begin{tabular}{|c|c|}
\hline \multirow{3}{*}{ 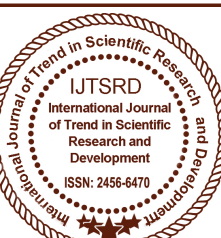 } & International Journal of Trend in Scientific Research and Development (IJTSRD) \\
\hline & International Open Access Journal | www.ijtsrd.com \\
\hline & ISSN No: 2456 - 6470 | Volume - 2 | Issue - 6 | Sep - Oct 2018 \\
\hline
\end{tabular}

\title{
A Review on Rain Fade and Signal Attenuation by Rain in Ku and Ka Band Satellite Communication at MGC Campus Punjab India
}

\author{
Er. Sukhjinder Singh ${ }^{1}$, Er. Iqbal Singh ${ }^{2}$, Er. Jasraj Singh ${ }^{3}$ \\ Assistant professor \\ ${ }^{1}$ Head of Department EEE, ${ }^{2}$ Head of Department CSE, ${ }^{3}$ Head of Department EE, \\ Modern Group of Colleges, Mukerian, Punjab, India
}

\begin{abstract}
Rain attenuation is a major challenge to microwave satellite communication especially at frequencies above $10 \mathrm{GHz}$, causing unavailability of signals most of the time. Rain attenuation predictions have become one of the vital considerations while setting up a satellite communication link.

Performance of the wireless communication system depends on the transmission path between source and destination (transmitter and receiver) which is extremely random, vary significantly over different frequency band. because of the heavy rainfall, snowfall, and other climatic condition most of the time wireless communication links are out of service and hence the performance of transmission of microwave signal from source to destination attenuate a lot, our primary concern is here outage due to rain. Attenuation because of rainfall play a significant role in design of terrestrial and Earth-satellite radio link specially at frequencies above $10 \mathrm{GHz}$. Therefore to design a link channel our major concern is to evaluate attenuation due to rainfall.
\end{abstract}

Keyword: RAIN FADE, INTELSAT, BER, KU, KA, X, $C, K, S, L, B A N D$

\section{INTRODUCTION}

In the frequency range above $10 \mathrm{GHz}$, rainfall becomes a serious and major source of attenuation for microwave communication [1]. Atmospheric effects play a major role in designing terrestrial and satellite to-Earth links operating at frequencies above $10 \mathrm{GHz}$.

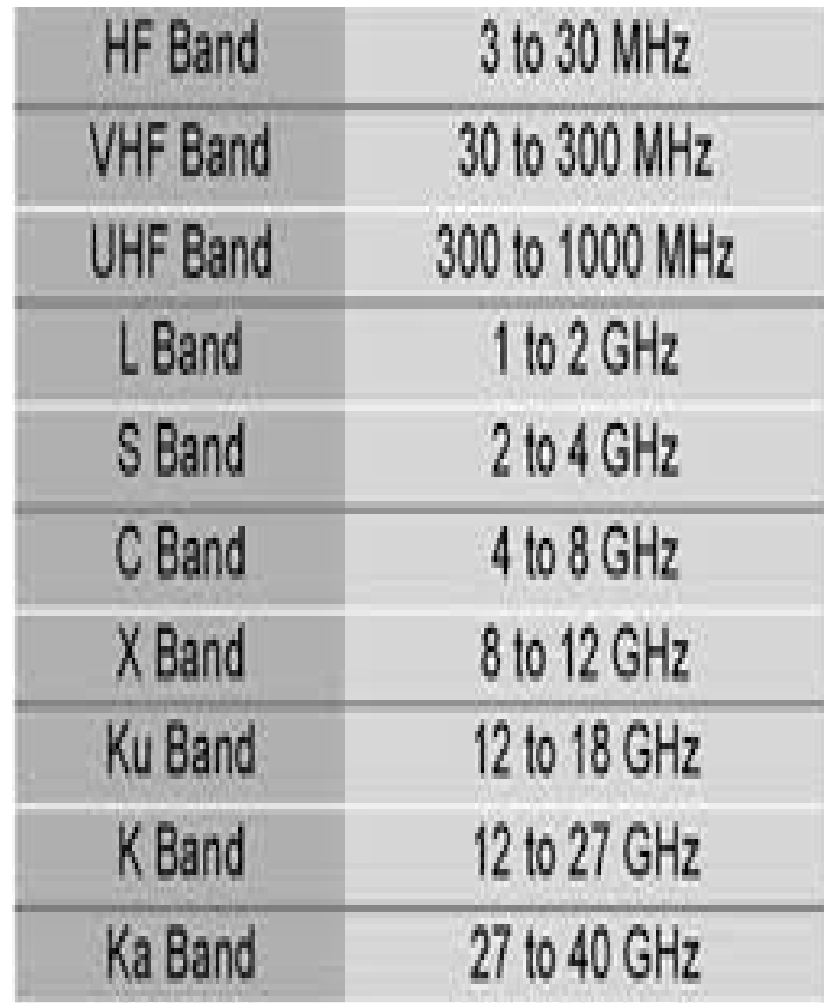

Figure 1: frequency range of various band

Raindrops absorb and scatter radio waves, leading to signal attenuation, and the reduction of the availability and reliability of systems. The severity of rain impairment increases with frequency, and varies with regional location [2]. The incidence of rainfall over radio links hence becomes even more important for frequencies as low as about $5 \mathrm{GHz}$, particularly in the tropical and equatorial climates, where intense rainfall events are common [3]. 


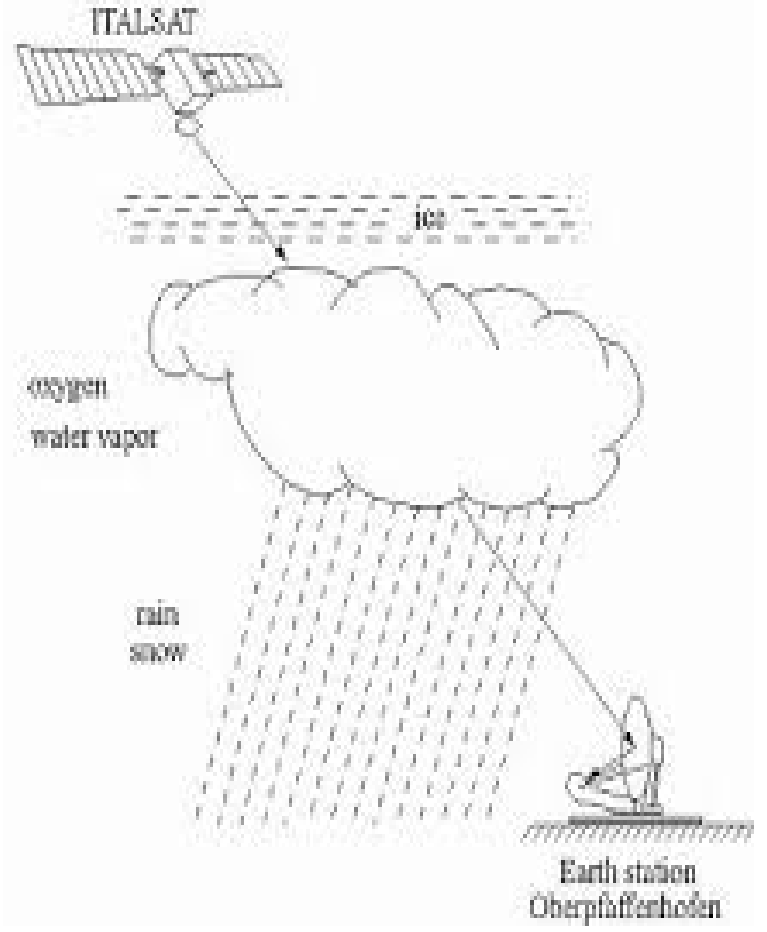

Figure2. ku \& ka band effected by rain

It is therefore very important when planning both microwave and terrestrial line-of-sight system links to make an accurate prediction of rain-induced attenuation over propagation paths. Initially, attenuation-prediction attempts involved extrapolation of measurements to other locations, frequencies, and elevation angles. However, the complex nature and regional variability of rain makes this approach highly inaccurate.

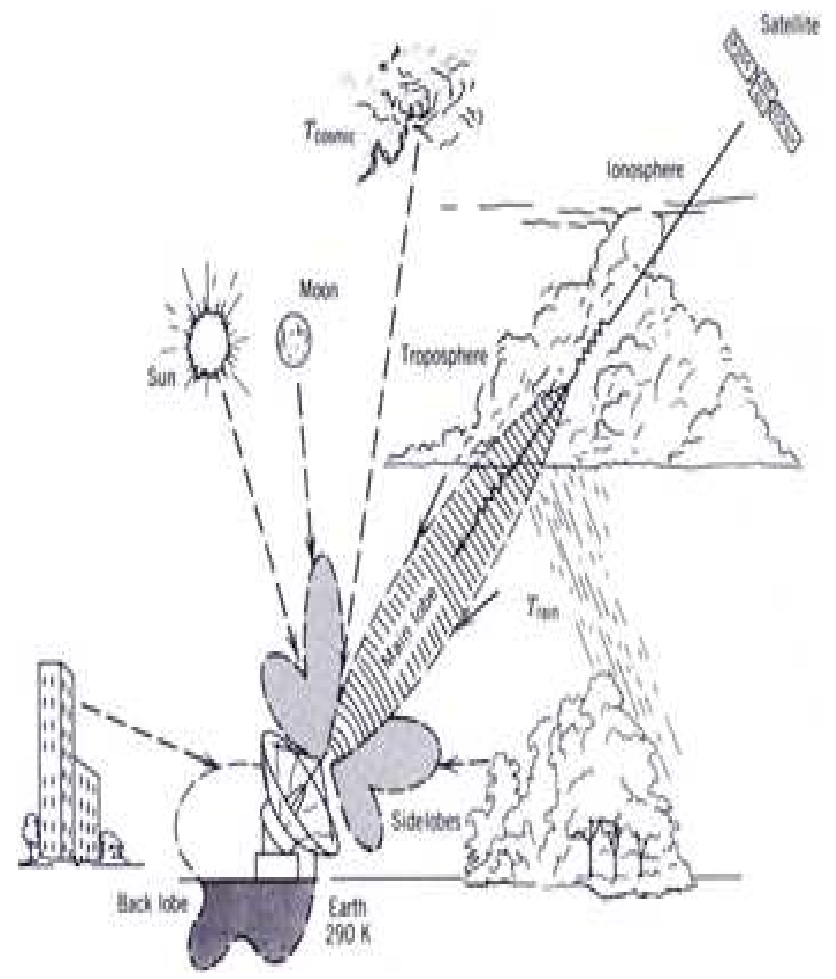

Figure 3: factor effecting ku and ka band
Figure 3 show factor effecting ku and ka band signal, rain , sun light, clouds, buildings and obstacles Performance of the satellite communication system depends on the transmission path between source and destination (transmitter and receiver) which is extremely random, vary significantly over different frequency band. In because of the heavy rainfall, snowfall, and other climatic condition most of the time wireless communication links are out of service and hence the performance of transmission of microwave signal from source to destination attenuate a lot, our primary concern is here outage due to rain. Attenuation because of rainfall play a significant role in design of terrestrial and Earth-satellite radio link specially at frequencies above $10 \mathrm{GHz}$. Therefore to design a link channel our major concern is to evaluate attenuation due to rainfall. Figure 3 show the rain fade in $\mathrm{ku}$ and $\mathrm{ka}$ band satellite communication

\section{RAIN FADE}

Rain fade refers primarily to the absorption of a microwave radio frequency (RF) signal by atmospheric rain, snow, or ice, and losses which are especially prevalent at frequencies above $11 \mathrm{GHz}$. It also refers to the degradation of a signal caused by the electromagnetic interference of the leading edge of a storm front. Rain fade can be caused by precipitation at the uplink or downlink location. However, it does not need to be raining at a location for it to be affected by rain fade, as the signal may pass through precipitation many miles away, especially if the satellite dish has a low look angle. From 5 to 20 percent of rain fade or satellite signal attenuation may also be caused by rain, snow, or ice on the uplink or downlink antenna reflector, radome or feed horn. Rain fade is not limited to satellite uplinks or downlinks; it also can affect terrestrial point to point microwave links (those on the earth's surface).

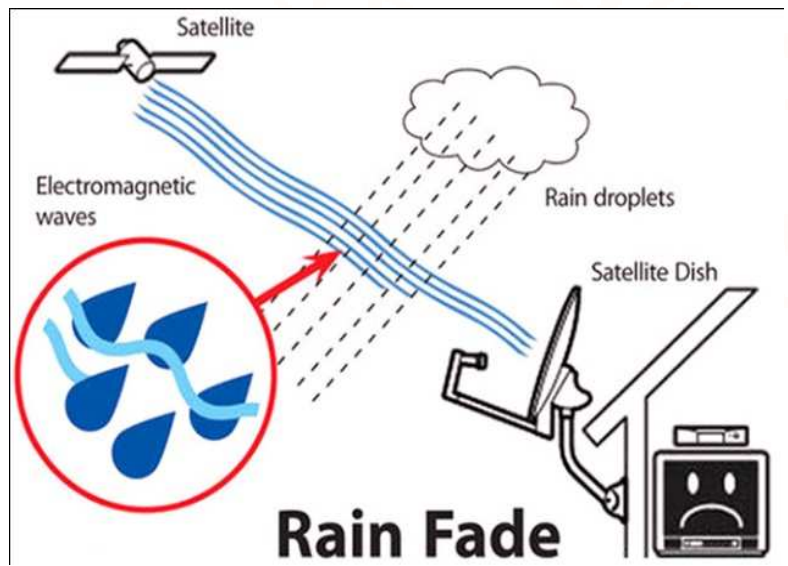

Figure 4: rain fade 
For radio path engineering, a procedure is needed to calculate the rain attenuation distribution on mill metric radio paths from the available rainfall data. There are two approaches to predict the rain attenuation, a physical method in which rain is described all the way along the path, and in empirical method which uses the effective path length

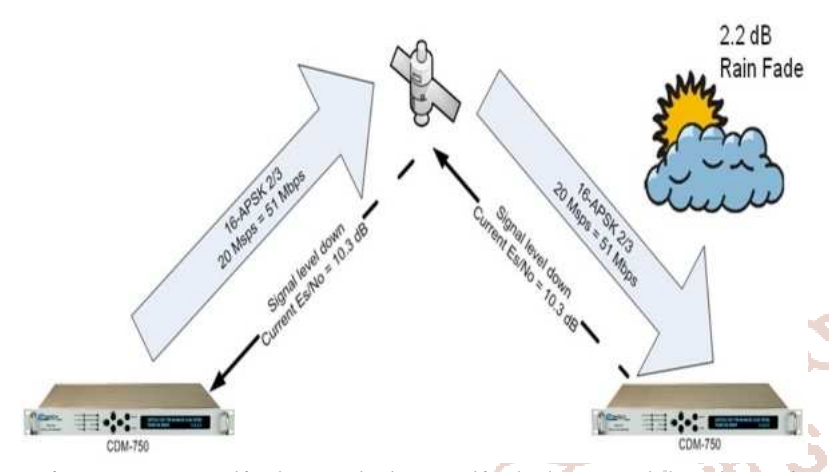

Figure 5: uplink and downlink ka and ku band

Figure 5 show how fade in uplinking and downlinking effect ku and ka band.

Rain fade is usually estimated experimentally and also can be calculated theoretically using scattering theory of rain drops. Raindrop size distribution (DSD) is an important consideration for studying rain fade characteristics. Various mathematical forms such as Gamma function, lognormal or exponential forms are usually used to model the DSD. Mie or Rayleigh scattering theory with point matching or t-matrix approach is used to calculate the scattering cross section, and specific rain attenuation. Since rain is a non-homogeneous process in both time and space, specific attenuation varies with location, time and rain type.

Total rain attenuation is also dependent upon the spatial structure of rain field. Horizontal as well vertical extension of rain again varies for different rain type and location. Limit of the vertical rain region is usually assumed to coincide with 0 degree isotherm and called rain height. Melting layer height is also used as the limits of rain region and can be estimated from the bright band signature of radar reflectivity. ${ }^{[2]}$ The horizontal rain structure is assumed to have a cellular form, called rain cell. Rain cell sizes can vary from a few hundred meters to several kilometers and dependent upon the rain type and location. Existence of very small size rain cells are recently observed in tropical rain. ${ }^{[3]}$
Possible ways to overcome the effects of rain fade are site diversity, uplink power control, variable rate encoding, and receiving antennas larger than the requested size for normal weather conditions.

Simply can be obtained from ITU-R. $\mathrm{R}$ is the rainfall rate in $\mathrm{mm} / \mathrm{hr}$. ITU-R model does not perform well in tropical climate region and in heavy rainfall region because the ITU-R based model is based on data collected from temperature region of world and provide good estimation for microwave loss caused by rain for temperature region but underestimate the prediction for tropical region. The rain drops absorb most of electromagnetic energy at Microwave $(>8 \mathrm{GHz})$

Hence, a regional rain map has to be developed to enable accurate radio link design at millimetre wave lengths, where attenuation due to rain is a major source of propagation outage. The values for rain attenuation are calculated for different rainfall rate using Simple Attenuation experiment will provide useful results for estimation of rainfall attenuation on microwave links in tropical region.

\section{CONCLUSION}

The simplest way to compensate the rain fade effect in satellite communications is to increase the transmission power: this dynamic fade countermeasure is called uplink power control (UPC). Until more recently, uplink power control had a limited use since it required more powerful transmitters - ones that could normally run at lower levels and could be run up in power level on command (i.e. automatically). Also uplink power control could not provide very large signal margins without compressing the transmitting amplifier. Modern amplifiers coupled with advanced uplink power control systems that offer automatic controls to prevent transponder saturation make uplink power control systems an effective, affordable and easy solution to rain fade in satellite signals. Rain attenuation is caused as a result of absorption of part or all of the signal's radiation power by the raindrop. This absorption is as a result of scattering effect (diffraction and refraction) of the rain drop to the signal. Rain attenuation is prevalent at frequencies above $10 \mathrm{GHz}$ and increases with frequency. This is because as frequency increases, the signal wavelength decreases and approaches the size of a rain drop and hence giving the rain drop more scattering and absorption capabilities to the signal. At a particular 
frequency, rain attenuation increases exponentially with increasing rain rate values because, the higher the rainfall rate, the higher the rain drop size (rain drop diameter). Hence, it is more its scattering effect on the signal, because as the rain drop size increases, it tends to approach the wavelength of the signal.

\section{REFERENCES}

1. Stallins W., Data and Computer Communications. Upper Saddle River, New Jersey, United States, Pearson prentice hall, 2007.

2. Laplante P. A., IEEE Electrical Engineering dictionary, N-W Boca Raton, CRC press ILC, 2000.

3. Rain Fade. Retrieved from http://en.wikipedia.org/ wiki/Rain_fade

4. Bryant H., Adinula I., Riva C. and Brussand G., Rain Attenuation Statistic from Rain Cell Diameters, and Heights. Int. J. Satell. Commun., 19, 2001, 263-283.

5. Mc Cormick K. S., Rain Attenuation Measurements in South East Asia. Moscow, Dompars, June 1994.

6. Propagation in non Lonized media. CCIR, Vol. V, Geneva 1990.

7. Barclay P. A., Benett J. A. and Boston R. C., Properties of Rain for Microwave Propagation Studies. Report from the Electrical Engineering Department, School of Engineering, Monash University, Melbourne (Australia), 1978.

8. Akeyama A., Morita K., 11 and $18 \mathrm{GHz}$ Radiowave Attenuation due to Precipitation on a Slant Path. IEEE Transaction Antennas and Propagation, Vol. AP-28(4), 1980, 580-585.

9. Moupfouma F. L, Martin N., Rain Structure on Attenuation due to Precipitation based on Measurement in Tropical Zone. 3-7 December, 1990.
10. Moupfouma F., Rainfall Rate Cumulative Distribution Modelling for the Determination of Rain Attenuation Statistics in Terrestrial and Satellite Radio Links in Tropical areas. Seminar on Radiowave Propagation in Tropical Regions, Triete (Italy), 1993.

11. Facts about Rain Fade and Satellites. http://www. phonetvinternet.com

12. Laws J. O. and Parsons D. A., The Relation of Raindrop sizes to Intensity. Trans Amer. 138 Geophys. Union, Vol. 24, 1943, 432-460.

13. Marshall J. S. and Palmer W. M. K. The Distribution of Raindrops with size. Journal of Meteorology, Vol. 5, 1948, 165-166.

14. Joss J., Thams J. C. and Waldvogel A., The Variation of Raindrop Size Distribution at Lorcano. Switzerland, Proc. Int. Conf, on Cloud Physics Toronto (Canada), 1968, 369-373.

15. Ajewole M. O., Scattering and Attenuation of Centimeter and Millimeter Radio Signals by Tropical Rainfall. Ph.D thesis, Federal University of Technology Akure Nigeria (unpublished), 1997.

16. Availability. Retrieved from http://en.wikipedia. org/wiki/Availability

17. Ojo J. S. and Ajewole M. O., Rain Rate and Rain Attenuation Prediction for Satellite Communication in $\mathrm{Ku}$ and $\mathrm{Ka}$ bands over Nigeria. Progress in Electromagnetic Research B, Vol. 5, 2008, 207-223.

18. Ajayi G. O., Slant Path Rain Attenuation measurement in Africa. Seminar on Radio wave Propagation in Tropical Regions, Trieste (Italy), 1993.

19. Alnutt J. E. and Rogers D. V., The INTELSAT Slant Path Radio wave Propagation Experiment in the Tropical 